Mens

revue d'histoire intellectuelle de l'Amérique française

\title{
V. Une crise intellectuelle (1929-1945)
}

Volume 6, numéro supplément, 2005-2006

URI : https://id.erudit.org/iderudit/1024240ar

DOI : https://doi.org/10.7202/1024240ar

Aller au sommaire du numéro

Éditeur(s)

Centre de recherche en civilisation canadienne-française

ISSN

1492-8647 (imprimé)

1927-9299 (numérique)

Découvrir la revue

Citer ce document

(2005). V. Une crise intellectuelle (1929-1945). Mens, 6, 63-75.

https://doi.org/10.7202/1024240ar d'utilisation que vous pouvez consulter en ligne.

https://apropos.erudit.org/fr/usagers/politique-dutilisation/ 
VOISINE, Nive, "Louis Gonnet ", Dictionnaire biographique du Canada, XIV (de 1911 à 1920), Québec, Presses de l'Université Laval, 1998, p. 451-453. http://www.biographi.ca.

WARREN, Jean-Philippe, "La découverte de la "question sociale". Sociologie et mouvements d'action jeunesse canadiensfrançais ", Relue d'bistoire de l'Amérique française, 55, 4 (printemps 2002), p. 539-572.

WARREN, Jean-Philippe, L'engagement sociologique : la tradition sociologique du Québec francophone (1886-1955), Montréal, Boréal, 2003, 448 p.

WARREN, Jean-Philippe, "Inquiétudes et espoirs au Québec en 1900 », Argument, 6, 1 (automne 2003-hiver 2004), p. 124-132.

WILLIS, John, "L'importance sociale du bureau de poste en milieu rural au Canada (1880-1945)", Histoire sociale/Social History, 30, 59 (mai 1997), p. 143-168.

\section{Une crise intellectuelle (1929-1945)}

ALMIEIDA, Mike, L'office protincial des recherches scientifiques et le déreloppement de la sicience au Québec, 1937-1960, M.A. (Histoire), Université du Québec à Montréal, 2002, 76 p.

AMYOT, Éric, La bataille pour le Québec: Vichy. La France libre et les Canadiens français (1940-1945), Ph.D. (Histoire), Université MIcGill, 1998, 452 p.

AMYOT, Éric, Le Québec entre Pétain et de Gaulle : Vichy, la France libre et les Canadiens français, 1940-1945, Saint-Laurent, Fides, 1999, $365 \mathrm{p}$.

AMYOT, Éric, «Vichy, la France libre et le Canada français : bilan historiographique », Bulletin d'bistoire politique, 7, 2 (hiver 1999), p. 917.

ANCTIL, Pierre, "Réponse à Esther Delisle sur l'antisémitisme ", dans André Champagne (dir.), Le Québec contemporain, Sillery, Septentrion, 1995, p. 55-68. 
ANGERS-FABRE, Stéphanie, "Le versant canadien-français de la génération non-conformiste européenne des années trente : la revue La Relère ", Recheribes sociographiques, 43, 1 (2002), p. 133-148.

BARRIS, Alex, Oscar Peterson: A Musical Biography, Scarborough, Harper Collins Canada, 2002, 256 p.

BEAUREGARD, Claude, Guerre et censure au Canada (1939-1945), Sillery, Septentrion, 1998, 196 p.

BEAUREGARD, Claude, «La propagande de Vichy et la réponse du gouvernement canadien », Bulletin d'histoire politique, 7, 2 (hiver 1999), p. 33-42.

BÉGIN, Yves, « Nationalisme et antinationalisme chez JeanCharles Harvey (1935-1939) ", dans Troucer notre place dans le monde/ Finding Our Place in the World, Documents de travail de l'Institut d'études canadiennes de McGill, Association des étudiantes et étudiants de 2 e et 3 e cycles en études canadiennes de McGill, sixième colloque annuel, 8-9 mars 2001, Université MicGill, p. 3546.

BÉGIN, Yves, Raison et sentiment : nationalisme et antinationalisme dans Le Québec des années 1935-1939, M.A. (Histoire), Université de Montréal, 2001, vi-144 p.

BÉGIN, Yves, "Sur Nationalisme et religion (1936) de Louis Lachance, o.p. ", Mens. Rei'ue d'bistoire intellectuelle de l'Amérique française, 2, 2 (printemps 2002), p. 155-192.

BEHIELS, Michael D., "Georges-Henri Lévesque: Father of Quebec's Lucid Integration into Confederation, 1930-1962", dans Jean-Pierre Wallot (dir.), avec la collaboration de Pierre Lanthier et Hubert Watelet, Constructions identitaires et pratiques sociales : actes $d u$ colloque en hommage à Pierre Saiard, tenu à l'Université d'Ottawa les 4, 5, 6 octobre 2000, Ottawa, Les Presses de l'Université d'Ottawa, 2002, p. $105-123$.

BÉLANGER, Damien-Claude, "Lionel Groulx et la crise sentinelliste ", Mens. Reı'ue d'histoire intellectuelle de l'A mérique française, 1, 1 (automne 2000), p. 7-36. 
BÉLANGER, Damien-Claude, Lionel Groulx et la FrancoAméricanie, M.A. (Histoire), Université de Montréal, 2000, vii$184 \mathrm{p}$.

BERGERON, Liette, Entre tradition et modernité : le chassé-croisé éditorial d'Albert Lévesque, Ph.D. (Littérature), Université de Sherbrooke, 2001, 489 p.

BERGERON, Marco, Le nationalisme et les partis politiques dans l'élection prorinciale québécoise de 1936, M.A. (Histoire), Université Laval, 1998, 112 p.

BÉRUBÉ, Harold, Commémorer la iille, une analyse comparative des célébrations du centenaire de Toronto en 1934 et du trientenaire de Montréal en 1942, M.A. (Histoire), Université de Montréal, 2002, viii-166 p. BIENVENUE, Louise, Une jeunesse en gestation. Les mourements de la jeunesse d'Action catholique spécialisée et l'affirmation de la jeunesse comme catégorie sociale au Québec (1930-1950), Ph.D. (Histoire), Université du Québec à Montréal, 1999, 381 p.

BIENVENUE, Louise, Quand la jeunesse entre en siène : l'action catholique arant la Rérolution tranquille, Montréal, Boréal, 2003, 291 p.

BOILY, Frédéric, «Un pétainiste convaincu : le chanoine Lionel Groulx », Cité Libre, 26, 2 (avril/mai 1998), p. 45-50.

BOILY, Frédéric, "Une figure du catholicisme social de l'entredeux-guerres : le père Joseph-Papin Archambault, s.j. ", Mens. Reı'ue d'histoire intellectuelle de l'Amérique française, 1, 2 (printemps 2000), p. 141-162.

BOILY, Frédéric, Naissance et croissance d'une "nourelle race " : les présupposés philosophiques, théologiques, politiques et sociaux du nationalisme de Lionel Grouli; Ph.D. (Sciences politiques), Université Laval, 2000, 265 p.

BOILY, Frédéric, La pensée nationaliste de Lionel Groulx, Sillery, Septentrion, 2003, 229 p. 
BOUCHER, Jean-Pierre, "Albert Laberge, contestataire avant l'heure ", Globe. Rène internationale d'études québécoises, 3, 1 (2000), p. 137-157.

BOURGEOIS, Patrick, Michel Chartrand : l'érolution idéologique d'un contestataire québécois : 1936-2000 : un parcours intellectuel marginal?, M.A. (Histoire), Université Laval, 173 p.

BROSSEAU, Louis, Le cinéma d'une guerre oubliée, Montréal, VLB éditeur, 1998, $205 \mathrm{p}$.

BRUNET, Jean-M Iarc, Le prophète solitaire. Raymond Barbeau et son époque, [Montréal], Ordre naturiste social de Saint-Marc l'Évangéliste Inc., 2000, $582 \mathrm{p}$.

CANUEL, Alain, "La censure en temps de guerre : RadioCanada et le plébiscite de 1942 ", Relue d'bistoire de l'Amérique française, 52, 2 (automne 1998), p. 217-242.

CANUEL, Alain, "Les avatars de la radio publique d'expression française au Canada, 1932-1939 ", Rel'ue d'bistoire de l'Amérique française, 51, 3 (hiver 1998), p. 327-356.

CANUEL, Alain, "Censure et politique en temps de guerre au Canada : le réseau public d'expression française », Bulletin d'histoire politique, 8, 2-3 (hiver-printemps 2000), p. 172-183.

CAREL, Ivan, "Le Nourelliste : un quotidien régional et la tourmente française ", Bulletin d'bistoire politique, 7, 2 (hiver 1999), p. 1832.

CLOUTIER, Yvan, "Légitimité de l'apostolat de l'édition : Le Lévrier et Fides ", Études d'histoire religieuse/Historical Studies. The Canadian Catholic Historical Association, 67 (2001), p. 271-280.

COMIMIEND, Susanne, De la femme déchue à la femme infectieuse : perieption sociale et répressions de la prostitution montréalaise pendant la seconde guerre, M.A. (Histoire), Université de Montréal, 1997, 169 p.

DAGENAIS, Michèle, "Le Jardin botanique de Montréal : une responsabilité municipale? ", Rel'ue d'bistoire de l'Amérique française, 52, 1 (été 1998), p. 3-22. 
DELISLE, Esther, "Lionel Groulx et l'antisémitisme », dans André Champagne (dir.), Le Québec contemporain, Sillery, Septentrion, 1995, p. 41-54.

DÉSY, Caroline, Discours hégémonique et contre-discours sur la guerre d'Espagne dans le Québec des années trente, Ph.D. (Sociologie), Université du Québec à Montréal, 1999, 292 p.

DÉSY, Caroline, « Fascisme et anti-fascisme autour de la guerre civile espagnole dans le Québec des années trente ", Bulletin d'histoire politique, 9, 2 (hiver 2001), p. 30-39.

DUBÉ, Ludwig, Images et perieptions de la jeunesse étudiante universitaire québécoise à traiers les journaux étudiants (1930-1939), M.A. (Histoire), Université du Québec à Montréal, 1999, 124 p.

DUCHESNE, Pierre, Jacques Parizeau, Biographie, 1930-1970, Tome 1: Le Croisé, Montréal, Québec Amérique, 2001, 624 p.

DURFLINGER, Serge M., City at War: the Effects of the Second World War on I'erdun, Québec, Ph.D. (Histoire), M IcGill University, 1997, 467 p.

FABRE, Julien, La Nation : les groulizens derant la tentation fasciste (1936-1939), M.A. (Histoire), Université Pierre Mendès-France, Grenoble, 1999.

FABRE, Julien, «La Nation: les groulxiens devant la tentation fasciste 1936-1939 », Bulletin d'bistoire politique, 9, 2 (hiver 2001), p. 40-51.

FERRET"TI, Lucia, "Les agences sociales à Montréal, 19321971 ", Études d'histoire religieuse/Historical Studies. The Canadian Catholic Historical Association, 66 (2000), p. 69-87.

FERRETTI, Lucia, "Charles-Édouard Bourgeois, prêtre trifluvien, et les origines diocésaines de l'État-providence au Québec (1930-1960) ", Nourelles pratiques sociales, 14, 1 (juin 2001), p. $169-182$.

GAGNON, François-Marc, Cbronique du mourement automatiste québécois (1941-1954), Outremont, Lanctôt Éditeur, 1998, 1023 p. 
GAGNON, Jérôme, "Les débuts du cinéma au Saguenay-LacSaint-Jean : le tournage du film Maria Chapdelaine en 1934 », Saguenayensia, 42, 2 (2000), p. 29-35.

GAGNON, Robert, "La Seconde Guerre mondiale et l'émergence de la recherche », dans Serge Bernier (dir.), L'impait de la Deuxième Guerre mondiale sur les sociétés canadienne et québécoise, Ottawa, Défense nationale, 1998, p. 141-158.

GARIÉPY, Alain, Jean-Marc Léger: un intellectuel nationaliste du Québec contemporain, M.A. (Histoire) Université Laval, 1996, 122 p.

GAUVREAU, Michael, «From Rechristianization to Contestation: Catholic Values and Quebec Society, 1931-1970 ", Church History, 69, 4 (décembre 2000), p. 803-833.

GAUVREAU, Michael, "The Emergence of Personalist Feminism: Catholicism and the Marriage-Preparation Movement in Quebec (1940-1966)", In Nancy Christie (dir.), Households of Faith: Family, Gender, and Community in Canada, 1760-1969, Montréal et Kingston, McGill-Queen's University Press, 2002, p. 319347.

GERVAIS, Diane et Danielle GAUVREAU, «Women, Priests, and Physicians: Family Limitation in Quebec, 1940-1970 », Journal of Interdisciplinary History, 34, 2 (automne 2003), p. 293-314.

GINGRAS, Anne-M Iarie, "Éthique et argument ad populum dans les débats télévisés canadiens (1962-1997) », Communication : information, médias, théories, pratiques, 18, 2 (1998), p. 53-69.

GINGRAS, Chantale, I iitor Barbeau. Un réseau d'influences littéraires, Montréal, L'Hexagone, 2000, 212 p.

GINGRAS, Yves, "Le rôle d'intellectuel des scientifiques québécois ", dans Manon Brunet et Pierre Lanthier (dirs.), L'inscription sociale de l'intellectuel, Sainte-Foy/Paris, Les Presses de l'Université Laval/L'Harmattan, 2000, 331-340.

GUILLAUME, Sylvie, "L'américanité des observateurs québécois de la vie politique française, des années trente aux années soixante ", Études canadiennes/Canadian Studies, 43 (1997), p. 61-74. 
HÉBERT, Pierre et Marie-Pier LUNEAU, «L'écrivain conscrit : la Seconde Guerre mondiale, la censure et les positions de trois écrivains québécois ", dans Manon Brunet et Pierre Lanthier (dirs.), L'insiription sociale de l'intellectuel, Sainte-Foy/Paris, Les Presses de l'Université Laval/L'Harmattan, 2000, p. 233-244.

HELLMAN, John, " Monasteries, Miliciens, War Criminals: Vichy France/Québec (1940-1950) », Journal of Contemporary History, 32, 4 (octobre 1997), p. 539-554.

HOULE, Nancy, "La Relère : une revue, un réseau », dans Pierre Rajotte (dir.), Lieux et réseaux de sociabilité littéraire au Québec, Québec, Éditions Nota bene, 2001, p. 113-153.

JEDWAB, Jack, « Notre maitre : le passé ? Nationalisme et antisémitisme au Deroir (1932-1947) », dans Robert Comeau et Luc Desrochers (dirs.), Le Devoir : un journal indépendant (19101995), Montréal, Les Presses de l'Université du Québec, 1996, p. 199-210.

LABERGE, Suzanne, "L'affaire Richard/Campbell : un catalyseur de l'affirmation des Canadiens français ", Bulletin d'bistoire politique, 11, 2 (hiver 2003), p. 30-44.

LACHANCE, Micheline, Paul-Émile Léger, 2 vol. Tome 1: Le prince de l'Église, 406 p. ; Tome 2 : Le dernier voyage, 357 p. Montréal, Éditions de l'Homme, 2000.

LANOUETTE, Mélanie, Faire ivire ou faire connaitre: Les défis de l'enseignement religieux en contexte de renoureau pédagogique, 1936-1946, Sainte-Foy, Les Presses de l'Université Laval, 2002, 174 p.

LAPOINTE, Mathieu, Nationalisme et socialisme dans la pensée de Raoul Roy (1935-1965), M.A. (Histoire), Université de Montréal, 2002, viii-212 p.

LAVERTU, Yves, Jean-Charles Hariey, le combattant, Montréal, Boréal, 2000, 462 p.

LEFEBVRE, Marie-Thérèse, "Les soirées Mathieu (1930-1935) ", Les Cahiers des Dix; 57 (2003), p. 85-118. 
LEGRIS, Renée, «L'institution ecclésiale et les structures de l'idéologie chrétienne dans les radioromans et les dramatisations historiques (1935-1975) ", Études d'histoire religieuse/Historical Studies. The Canadian Catholic Historical Association, 68 (2002), p. 41-56.

LEROUX, Éric, « Des moyens de faire face : les syndicats internationaux et la crise des années trente ", Bulletin d'bistoire politique, 9, 2 (hiver 2001), p. 73-83.

LEROUX, Éric, «Les projets de société de la FPTQ et de la FUIQ (1938-1957) : ressemblances et différences », dans Yves Bélanger, Robert Comeau et Céline Métivier (dirs.), La FTQ, ses syndicats et la société québécoise, Montréal, Comeau \& Nadeau, 2001, p. 27-53.

LEVERT, Myriam, La censure de la presse d'expression française du Québec durant la Première Guerre mondiale, M.A. (Histoire), Université du Québec à Montréal, 2001, iv-194 p.

LÉVESQUE, Andrée, «Anniversaires et manifestations des camarades : la culture internationale et l'identitaire communistes au Canada pendant l'entre-deux-guerres ", Labour/Le Traiail, 49 (printemps 2002), p. 83-92.

LUNEAU, Marie-Pier, Le manche et la cognée : Lionel Groulx, une rie d'éritures, Ph.D. (Études françaises), Université de Sherbrooke, 2001, 486 p.

LUNEAU, Marie-Pier, Lionel Groulx : Le mythe du berger, Montréal, Leméac, 2003, 226 p.

MARCO'T"TE, Gilles, "Pauvreté d'Hector de Saint-Denys Garneau (1912-1943) ", dans Gilles ROUTHIER et Jean-Philippe WARREN, dir., Les Visages de la foi. Figures marquantes du catholicisme québéiois, Montréal, Fides, 2003, p. 115.

MLARTEL, Marcel, "André Laurendeau et les groupes francophones en milieu minoritaire (1935-1968) ", Les Cahiers d'histoire du Québec au XXe siècle, 10 (hiver 2000), p. 80-87. 
MLARTIN, M lichèle, «L'éducation des publics : la critique culturelle journalistique de Victor Barbeau ", Historical Studies in Eduiation/ Rerue d'histoire de l'éduiation, 8, 2 (1996), p. 182-198.

MARTIN, Nichèle et William J. BUXTON, « Monopoles du savoir ou critique culturelle journalistique ? Innis et Victor Barbeau discutent la presse, le national isme et les pratiques intellectuelles ", dans Charles R. Acland et William J. Buxton (dirs.), Harold Innis in the Nen Century: Reflections and Refractions, Montréal et Kingston, McGill-Queen's University Press, 1999, p. 159-174.

MARTIN, Michèle, et al, "La pré-modernité de Radiomonde : un pas hésitant vers un Québec moderne ", Histoire sociale/Soizal History, 33, 65 (2000), p. 37-57.

MATTHEWS, Mary, Working for Family, Nation and God: Paternalism and the Dupuis Frères Department Store. Montreal, 1926 1952, M.A. (Histoire), Université MicGill, 1998, 156 p.

MICHON, Jacques, Fides, La grande arenture éditoriale du père PaulAimé Martin, Montréal, Fides, 1998, 387 p.

MORISSET, Stéphane, Adrien Ariand : sa izision, son modèle et la perception inspirée par son programme, M.A. (Histoire), Université Laval, 1996, 86 p.

MORIN, Nadine, L'aulre des terrains de jeux; Sherbrooke (1929. 1962), M.A. (Histoire), Université de Sherbrooke, 2000, 117 p.

MURTON, James, "La "Normandie du Nouveau Monde" : la société Canada Steamship Lines, l'antimodernisme et la promotion du Québec ancien ", Rel'ue d'histoire de l'Amérique française, 55, 1 (été 2001), p. 3-44.

NURSE, Andrew, Tradition and Modernity: The Cultural Work, of Marius Barbeau, Ph.D. (Histoire), Queen's University, 1997, 632 p.

PAGÉ, Pierre, «Cinquante ans d'émissions religieuses à la radio québécoise (1931-1983). De l'apologétique au dialogue avec les grandes religions ", Études d'histoire religieuse/Historical Studies. The Canadian Catholic Historical Association, 68 (2002), p. 7-23. 
PANNETON, Jean-Charles, Georges-Émile Lapalme : un réformiste engagé (1930-1960), M.A. (Histoire), Université du Québec à Montréal, 1999, 151 p.

PERRAULT, Isabelle, Le plaisir sous surveillanie : analyse des discours iatholiques sur la sexualité conjugale et féminine dans les manuels de sexualité maritale au Québec (1930-1960), M.A. (Histoire), Université du Québec à Montréal, 2003, vii-125 p.

PERRON, Mathieu, Jaiques Labrecque : trajectoire d'un «diffuseur du folklore ", M.A. (Histoire), Université Laval, 2002, v-162 p.

PEYREFITTE, Alain, De Gaulle et le Québec; Montréal, Stanké, 2000, 184 p.

PICHÉ, Lucie, « La Jeunesse ouvrière catholique féminine. Un lieu de formation sociale et d'action communautaire (1931-1966)", Retue d'histoire de l'Amérique française, 52, 4 (printemps 1999), p. 481506.

PICHÉ, Lucie, « Une jeunesse unique ? Mouvements de jeunesse et contestation sociale ", Études d'histoire religieuse/Historical Studies. The Canadian Catholic Historical Association, 67 (2001), p. 215-226.

PICHÉ, Lucie, Femmes et changement social au Québec : l'apport de la Jeunesse ouirière catholique féminine (1931-1966), Québec, Les Presses de l'Université Laval, coll. « Religions, cultures et sociétés », 2003, xxii-349 $\mathrm{p}$.

POIRIER, Patrick, La représentation du régime hitlérien par les éditorialistes du quotidien "La Patrie » (1933-1939), M.A. (Histoire), Université du Québec à Montréal, 2000, 132 p.

POIRIER, Patrick, " La représentation du régime hitlérien par $\mathrm{La}$ Patrie de Montréal (1933-1939) ", Mens. Retue d'bistoire intellectuelle de l'Amérique française, 4, 1 (automne 2003), p. 69-93. 
POMEYROLS, Catherine, "Les représentations culturelles de l'espace canadien chez les nationalistes québécois : le thème de la Laurentie dans l'entre-deux-guerres ", dans Sylvie Guillaume et al. (dirs.), L'espace canadien et ses représentations, Actes du colloque international tenu à Talence les 16 et 17 décembre 1994, Éditions de la Maison des sciences de l'homme d'Aquitaine, p. 21-34.

POMEYROLS, Catherine, "Les intellectuels de l'entre-deuxguerres : les milieux de formation ", dans Manon Brunet et Pierre Lanthier (dirs.), L'insiription sociale de l'intellectuel, Sainte-Foy/Paris, Les Presses de l'Université Laval/L'Harmattan, 2000, p. 213-232.

POMEYROLS, Catherine, "Comment fait-on l'histoire des intellectuels au Québec? ", dans Michel Leymarie et Jean-François Sirinelli (dirs.), L'bistoire des intellectuels aujourd'hui, Paris, Presses universitaires de France, 2003, p. 107-122.

QUIRION, Lise, «La presse québécoise d'expression française face au procès du maréchal Pétain, 1945 ", Bulletin d'histoire politique, 7, 2 (hiver 1999), p. 43-58.

QUIRION, Lise, Le proiès du maréchal Pétain dans la presse québécoise d'expression française (juillet et aoùt 1945), M.A. (Histoire), Université du Québec à Montréal, 1998, 144 p.

RÉGIMBALD, Patrice, " La disciplinarisation de l'histoire au Canada français (1920-1950) ", Ret'ue d'histoire de l'Amérique française, 51, 2 (automne 1997), p. 163-200.

ROBITAILLE, Marie-Josée, Louis-Mariel Raymond, iritique de théatre et promoteur des écrivains français pendant la Deuxième Guerre mondiale et l'après-guerre, M.A. (Études françaises), Université de Sherbrooke, 2000, 149 p.

RUDIN, Ronald, Faire de l'histoire au Québec; Sillery, Septentrion, 1998, 278 p.

RUELLAND, Jacques G., avec la collaboration de Marie-Josée FLEURY, Philosopher à Montréal : histoire de la Société de Philosophie de Montréal depuis 1933, M Iontréal, Humanitas, coll. "Circonstances ", 1995, 91 p. 
RYAN, Pascale, Des intellectuels dans la cité : la Ligue d'action nationale (1933-1960), Ph.D. (Histoire), Université du Québec à Montréal, 2002, x-315 p.

RYAN, Pascale, «Des intellectuels en Europe et en Amérique : un état de la question ", Mens. Reche d'histoire intellectuelle de l'Amérique française, 4, 1 (automne 2003), p. 9-37.

SAINT-DENYS GARNEAU, Hector de, CEures en proses, édition critique établie par Giselle Huot, Saint-Laurent, Fides, 1994, $1183 \mathrm{p}$.

SAINT-DENYS GARNEAU, Hector de, L'Univers de Saint-Denys Garneau, le peintre, le critique, présentation de France Gascon, Montréal/Joliette, Boréal/M lusée d'art de Joliette, 2001, 111 p.

SALAÜN, Élise, La Chair triomphante : discours religieux, juridique et iritique sur l'érotisme dans le roman au Québec (1940-1959), M.A. (Études françaises), Université de Sherbrooke, 1996, 197 p.

SAVARD, Pierre, "Une jeunesse et son Église : les scouts-routiers. De la Crise à la Révolution tranquille ", Les Cahiers des Diג; 53 (1999), p.117-165.

SEXTON, Jenny-Louise, I a perception de la politique française durant l'ocupation par des intellectuels franco-montréalais (1940-1944), M.A. (Histoire), Université du Québec à Montréal, 1998, 99 p.

TALBOT, Emile J. «Litterature and Ideology in the Thirties: Fictional Representations of Communism in Quebec ", International Journal of Canadian Studies/Rer'ue internationale d'études canadiennes, 20 (automne 1999), p. 67-80.

THIBAULT, Daniel, Ms Desranleau, élêque de Sherbrooke (19411952), et le catholicisme social, M.A. (Histoire), Université de Montréal, 2002, 164 p.

TREMBBLAY, Micheline et Guy GAUDREAU, «Le régionalisme littéraire au Canada français : le point de vue de Harry Bemard ", Globe. Rerue internationale d'études québécoises, 5, 1 (automne 2002), p. 159-178. 
TRÉPANIER, Pierre, "Le maurrassisme au Canada français », Les Cabiers des Did; 53 (1999), p.167-233.

VANNUCCI, Simone, Rôle structurant des aitions de la Compagnie de Jésus sur la littérature et l'édition au Québec (1930-1960), Ph.D. (Lettres et communication), Université de Sherbrooke, 2003, 362 p.

VIAT'TE, Auguste, D'un monde à l'autre: journal d'un intellectuel jurassien au Québec (1939-1949), édité et présenté par Claude Hausser, Sainte-Foy/Paris/Courrendlin (Suisse), Presses de l'Université Laval/L'Harmattan/Éditions Communication Jurassienne et Européenne (CJE), 2001, 584 p.

VIGNEAULT, Louise, Identité et modernité dans l'art au Québec':

Borduas, Sullitan, Riopelle, M Iontréal, Hurtubise HMIH, coll. « Cahiers du Québec », 2002, 406 p.

VINCENT, Josée, «Faire voir pour faire (re)connaitre : le travail de promotion de la Société des écrivains canadiens de 1936 à 1960 », Voix et images, 27, 2 (hiver 2002), p. 254-269.

WELLS, Stéphanie, La irise dans la correspondance des années trente: lecture sociocritique de lettres d'Alfred Des Rochers, Alain Grandbois et Saint-Denys Garneau, M.A. (Études françaises), Université de Montréal, 1998, 157 p.

\section{Pour une pensée libre (1945-1960)}

AIRD, Robert, L'érolution du comique de scène au Québec francophone depuis 1945, M.A. (Histoire), Université du Québec à Montréal, 2002, 169 p.

ANGERS-FABRE, Stéphanie, "Le regard de la revue Esprit sur le Québec », Français et Québécois : Le regard de l'autre, colloque tenu à Paris les 7, 8 et 9 octobre 1999 sous la direction de Jean-Pierre Bardet, Paris, Centre de coopération interuniversitaire francoquébécoise, 1999, p. 3-11.

ANGERS-FABRE, Stéphanie, Esprit au Québec : les reiues comme recteurs des échanges intellectuels franio-québécois, Ph.D. (Sociologie), Université d'Aix-Marseille I, 2000, 367 p. 